\title{
«ES DIFÍCIL SER COMPRENDIDO». UNA LECTURA DE JGB $27^{1}$

\author{
«It is hard to be understood» A reading of JGB 27
}

\author{
Axel Pichler \\ Universidad de Stuttgart
}

\begin{abstract}
RESUMEN: En la investigación alemana sobre Nietzsche, se ha establecido en los últimos años una práctica de interpretación que se enfoca en la formulación exacta de los textos de Nietzsche. Por lo tanto se denomina textnahe Interpretation (interpretación textual). El presente artículo quiere exhibir esta práctica de interpretación mediante la interpretación de un único texto de Nietzsche - MBM 27 - y hacerla accesible a un público poco familiarizado con la investigación actual en lengua alemana. Para este propósito, después de la interpretación, serán expuestas las suposiciones que implícitamente guían esta práctica de interpretación y serán discutidas sus consecuencias.
\end{abstract}

Palabras claves: MBM 27 - interpretación textual - metodología de la interpretación

AвSTRACT: In German Nietzsche scholarship, an interpretation practice has established itself in recent years which is characterized by its orientation to the exact wording of Nietzsche's texts and is therefore also referred to as textnahe Interpretation (close reading). The present article would like to demonstrate this practice of interpretation by means of the interpretation of a single text of Nietzsche - BGE 27 - and thus make it accessible to an audience unfamiliar with current German-language research. For this purpose, following the interpretation its secretly guiding assumptions are uncovered and their consequences discussed.

Keywords: BGE 27 - close reading - methodology

1 Este artículo es una versión revisada de Axel Pichler, «Präsumtionen und Praktiken textnaher Forschung. Eine exemplarische Lektüre von JGB 27»: Nietzscheforschung 27 (2020), 281-302.

Agradezco a Juan Manuel Heredia (Buenos Aires) y Marina Silenzi (Basel) por sus comentarios críticos sobre mi español, que tiene la tendencia de oscilar entre el léxico callejero del castellano del Río de la Plata y la sintaxis del alemán. 
En la investigación nietzscheana de habla alemana se ha establecido en los últimos años una práctica de interpretación que se caracteriza por su orientación hacia la enunciación exacta de los textos de Nietzsche y que, por lo tanto, se denomina interpretación textual ${ }^{2}$. Este enfoque representa una reacción crítica frente a las pretensiones de sistematizar el pensamiento tan polifacético de Nietzsche ${ }^{3}$.

En la presente contribución se desea demostrar esta práctica de interpretación textual in actu tomando como objeto un único texto de Nietzsche para hacerla accesible a un público que no está familiarizado con la investigación actual en lengua alemana. En principio, cabe señalar que este paradigma de interpretación se entiende aquí como una práctica de interpretación. Las prácticas de interpretación difieren de las teorías de interpretación en que, por una parte, representan un procedimiento guiado por reglas (normas), como los métodos de interpretación que se derivan de las teorías de interpretación, pero, por otra parte, esas reglas (normas) no se derivan de axiomas ni existen de forma explícita, sino que representan la aplicación de un conocimiento implícito. En qué medida en que este conocimiento implícito pueda hacerse explícito es discutible y depende principalmente del concepto subyacente de las reglas (normas).

La calificación de la investigación orientada al texto como práctica de interpretación se debe, entre otras cosas, a que las interpretaciones textuales más recientes de Nietzsche en lengua alemana no se caracterizan ni por un método uniforme ni, hasta la fecha -con algunas excepciones- por una reflexión metodológica común ${ }^{4}$. Dichas interpretaciones, sin embargo, no sólo coinciden en su enfoque hacia la enunciación exacta de los textos de Nietzsche, sino que también se asemejan en sus formas de tratar los textos de Nietzsche ${ }^{5}$. Werner Stegmaier, que puede considerarse como el iniciador

2 Las siguientes monografías y antologías son representativas para la evolución actual de este enfoque interpretativo: Werner Stegmaier, Nietzsches Befreiung der Philosophie. Kontextuelle Interpretation des V. Buches der «Fröhlichen Wissenschaft», Berlín/Boston: De Gruyter 2012; Marcus A. Born (ed.), Klassiker Auslegen: Friedrich Nietzsche, «Jenseits von gut und Böse», Berlín/Boston: De Gruyter 2014; Axel Pichler, Philosophie als Text-Zur Darstellungsform der «Götzen-Dämmerung», Berlín/Boston: De Gruyter 2014; Jakob Dellinger, Situationen der Selbstbezüglichkeit. Studien zur Reflexivität kritischer Denk- und Schreibformen bei Friedrich Nietzsche, tesis doctoral, Universität Wien 2015; Katarina Grätz/Sebastian Kaufmann (ed.), Nietzsche zwischen Philosophie und Literatur: Von der Fröhlichen Wissenschaft zu Also sprach Zarathustra, Heidelberg: Winter 2017.

3 Werner Stegmaier, «Nach Montinari. Zur Nietzsche-Philologie»: Nietzsche-Studien 36 (2007), 80-94.

$4 \mathrm{Al}$ menos así parece si se entiende por método un procedimiento encajado en un contexto teórico sistemático, fijado en la secuencia de sus pasos y repetible en sus resultados.

5 A pesar de estas similitudes, que, con Wittgenstein, se podrían llamar parecidos de familia, 
principal del enfoque orientado a los textos, ha formulado la idea fundamental que subyace a esta práctica de interpretación. Se expresa en la demanda de que los «textos [de Nietzsche] deben ser tomados en serio en la forma que él los dio» ${ }^{6}$. Esta convicción se basa en la tesis según la cual las formas de los textos de Nietzsche son filosóficamente válidas, es decir, que determinan esencialmente su contenido filosófico ${ }^{7}$. Estas formas incluyen, entre otras, el tipo de texto, las figuras retóricas y los tropos, los modos de diseño de las figuras, pero también la puntuación y la tipografía ${ }^{8}$.

Dado que el presente ensayo pretende ejemplificar de forma directa la práctica de interpretación textual, me abstendré preceder su desarrollo con una exposición detallada de la teoría de la interpretación en la que se basa, así del método que se deriva de ella. En lugar de ello, y siguiendo la interpretación de un solo texto de Nietzsche, se expondrán y reconstruirán los presupuestos que guían implícitamente a la interpretación. Dicha tarea se ve facilitada por el hecho de que el texto escogido -JGB 27-goza de una extensión que permite una interpretación detallada en los marcos acotados de un ensayo, así como porque se trata de una pieza que ya ha sido examinada desde diversas perspectivas, ya que Más allá del Bien y del Mal es el libro de Nietzsche que se ha convertido en el centro de la investigación en los últimos años. No sólo hay numerosas introducciones e interpretaciones del libro ${ }^{9}$, sino que además se cuenta con el comentario de Andreas Urs Sommer (Sommer 2016) ${ }^{10}$, que tiene casi 1000 páginas. También es una de las obras de Nietzsche cuya génesis está ampliamente documentada y -gracias al trabajo de Beat Röllin ${ }^{11}$ (cf. Röllin 2013)- ha sido reconstruida en detalle. Consideramos que esta

también hay diferencias en las interpretaciones de Nietzsche de los autores que se asignan aquí a la práctica de la interpretación textual. Estas diferencias consisten, en particular, en la cantidad de textos de Nietzsche que se tienen realmente en cuenta en una interpretación, así como qué secciones de la obra de Nietzsche se relacionan entre sí y sobre la base de qué criterios de selección en el curso de la interpretación.

6 Stegmaier, Nietzsches Befreiung der Philosophie (cit.), 77.

7 Si asumimos -como lo hacemos aquí- que la forma determina el contenido semántico de un texto, sólo es heurísticamente posible distinguir entre la forma y el contenido.

8 Para el estado de la investigación sobre las formas del filosofar de Nietzsche véase Stegmaier, Nietzsches Befreiung der Philosophie (cit.); Pichler, Philosophie als Text (cit.), 108-123 y Claus Zittel, «Der Dialog als philosophische Form bei Nietzsche»: Nietzsche-Studien 45 (2017), 81-112.

9 Los estudios más recientes son Laurence Lampert, Nietzsche's Task. An Interpretation of Beyond Good and Evil, New Haven/London: Yale UP 2001; Christa Davis Acampora y Keith Ansell Pearson, Nietzsche's Beyond Good and Evil. A Reader's Guide, London/New York: Continuum, 2011 y Born, Klassiker Auslegen (cit.).

10 Andreas Urs Sommer, Kommentar zu Nietzsches Jenseits von Gut und Böse, Berlín/Boston: De Gruyter 2016 (= Historischer und kritischer Kommentar zu Friedrich Nietzsches Werken, vol. 5/1).

11 Beat Röllin, «Ein Fädchen um’s Druckmanuskript und fertig? Zur Werkgenese von Jenseits von Gut und Böse», en: Marcus A. Born y Axel Pichler (ed.) Texturen des Denkens. Nietzsches Inszenierung der Philosophie in Jenseits von Gut und Böse, Berlín/Boston: De Gruyter 2013, 47-67. 
base proporciona a lo siguiente un marco que permite destacar una serie de especificidades y claves de una interpretación textual dentro de los límites de espacio que impone un ensayo.

$$
\mathrm{II}^{12}
$$

JGB 27 es la cuarta sección ${ }^{13}$ de la «segunda sección principal» de Más allá del Bien y del Mal - cuyo subtítulo «Preludio de una filosofía del futuro» ha desencadenado numerosas especulaciones sobre si ya se trata en sí mismo de su realización ${ }^{14}$. La investigación ha añadido recientemente una nueva dimensión a esta cuestión, así como al tratamiento del libro en su conjunto al demostrar que Más allá del Bien y del Mal se caracteriza en particular por el hecho de que en él las diferentes posiciones filosóficas «no sólo son presentadas de forma teórica, sino que se realizan textualmente» ${ }^{15}$ al ser articuladas y presentadas por diferentes figuras, entre las que uno o más narradores en primera persona del singular y del plural tienen un papel dominante.

Si uno sigue el texto hasta la sección 27, que es el foco de interés aquí, surge la siguiente imagen: Ya en el prefacio se articula la esperanza «de que todo dogmatizar en la filosofía [...] no habría sido más que una noble niñería de principiantes», cuya base -como se insinúa poco después-puede haber sido «una superstición popular cualquiera procedente de tiempos inmemoriales [...], tal vez un juego de palabras cualquiera, una seducción por parte de la gramática o una temeraria generalización de hechos muy reducidos, muy personales, muy humanos, demasiado humanos» (JGB, Prefacio). Esto marca el impulso del libro, que por un lado está dedicado a una crítica personalizada ${ }^{16}$ de las diversas formas de «superstición popular»

12 A menos que se indique específicamente, todas las citas siguientes se han tomado de Friedrich Nietzsche, Más allá del Bien y del Mal, en: Nietzsche, Obras completas, ed. de Diego Sánchez Meca. vol. IV: Escritos de Madurez II, Madrid: tecnos, 283-437. Algunas de las citas fueron revisadas en base al original en alemán.

13 La designación de «sección〉 para las piezas individuales de texto numeradas de JGB es neutral en la atribución de género y sigue una sugerencia de Andreas Urs Sommer (Sommer, Kommentar (cit.), 38), y se vincula con la investigación reciente, que ha demostrado que la designación convencional del aforismo como un texto corto aislado pero en su ubicación intercambiable (y, por tanto, infratextualmente indeterminado), se aplica sólo en una medida limitada a los libros de Nietzsche, que se publicaron después de Zarathustra. Más en general, en cuanto a los libros de Nietzsche, la atribución de género no es en absoluto trivial, ya que, dependiendo de la clasificación, otras relaciones intra- e infratextuales ganan relevancia para la interpretación de las secciones individuales.

14 Una sinopsis de las posiciones de investigación pertinentes se encuentra en Marcus Andreas Born, «Perspektiven auf eine Philosophie der Zukunft in Jenseits von Gut und Böse», en: Born, Klassiker Auslegen (cit.), 1-16.

15 ibid., 7.

16 «Crítica personalizada» significa no sólo que Nietzsche argumenta en algunas partes de JGB 
con el fin de preparar e iniciar la liberación de ellas, pero por otro lado expone tanto la condicionalidad personal como también la gramatical-lingüística de todo filosofar e intenta sondear sus consecuencias y límites. La primera sección principal comienza con un análisis de los «prejuicios de los filósofos» que oscila entre la descripción, la crítica y la redefinición, entendiendo por «prejuicios» las diferentes formas de creencia que -según los textos de la sección principal- están condicionadas fisio-psicológicamente, pero que en su condicionalidad condicionante subyacen e impregnan al pensamiento en su conjunto ${ }^{17}$.

Sobre esta base se desarrollan formas alternativas de pensamiento que se diferencian de las criticadas, en particular en el hecho de que exhiben su propio estatus de condicionalidad fisio-psicológica, la cual, si no se expresa completamente en la reflexión, sí lo hace en la expresión narrativa. Lo mismo ocurre con las tres secciones de la segunda sección principal que precede a JGB 27. JGB 24 retoma la problematización de la «voluntad de saber» que ya había tenido lugar en la primera sección principal y la asocia con el problema del lenguaje, que ya se había tratado anteriormente, para mostrar, por un lado, cómo esos conceptos, ya identificados como problemáticos, sirven a la vida a pesar de todo y, por el otro, para sacar los conceptos discutidos de las oposiciones rígidas y diferenciarlos aún más. En JGB 25 se hace un llamamiento enfático a los «filósofos y amigos del conocimiento» para que se abstengan de toda forma de determinación dogmática «a causa de la verdad», y luego, criticando a filósofos como Spinoza o Giordano Bruno por las consecuencias personales de su dogmatismo, se presentan dos alternativas: la «veracidad»y un llamamiento, que recuerda a Epicuro, a retirarse a la soledad o a protegerse de la compulsión de la determinación mediante el uso de máscaras. JGB 26 contrarresta indirectamente la última de las propuestas presentadas en JGB 25 sobre la base de una distinción entre «hombre[s] excelso[s]» y el «hombre

con y contra figuras históricas explícitamente nombradas - es decir, ad hominem - sino también que tanto la posición criticada como la crítica están siempre ligadas a una persona. Esta persona puede ser un yo-narrador, un nosotros-narrador o «todos los filósofos» (JGB 1). Sobre la personalización del pensamiento de Nietzsche y su función, cf. Werner Stegmaier, Friedrich Nietzsche zur Einführung, Hamburg: Junius 2011, 107f. y Axel Pichler, «Prosopopeisches Denken. Eine textgenetische Lektüre der Vorrede zur Neuauflage der ,Fröhlichen Wissenschaft'», en: Grätz/Kaufmann, Zwischen Philosophie und Literatur (cit.), 29-73.

17 Como sugieren las sinopsis de las investigaciones anteriores sobre la primera sección principal de JGB, ésta no sigue una «línea narrativa o argumentativa continua» (Sommer, Kommentar (cit.), 74), lo que plantea la cuestión de los criterios alternativos según los cuales se compone. Siguiendo a Born, «Perspektiven» (cit.), se puede asumir que, en la primera sección principal, la linealidad es reemplazada por el contrapunto, en el curso del cual las diferentes posiciones filosóficas se presentan en forma personalizada o tipificada. 
medio» al recomendar a los miembros del primer grupo el «estudio del hombre medio».

JGB 27 amplía en su primera oración la problemática lingüístico-filosófica ya desarrollada anteriormente en el libro en torno a, como se podría decir en la terminología actual, una cuestión hermenéutica-pragmatista. Mientras que las secciones anteriores, que ya tratan preguntas de la filosofía del lenguaje, se centran en particular en las consecuencias de determinadas estructuras gramaticales y en sus condiciones fisiológicas previas para filosofar (cf. JGB $16,17,20,34)$, el texto dice ahora, sin ninguna referencia explícita a las secciones anteriores ${ }^{18}$ : «Es difícil ser comprendido» ${ }^{19}$.

La frase implica una concepción del lenguaje o de la comunicación según la cual es posible, en principio, ser comprendido, pero esta comprensión no está en absoluto garantizada ${ }^{20}$, aunque en este punto del texto no se explicita todavía si una falta de comprensión conduce a un no-entendimiento absoluto o a una malinterpretación. También es sorprendente que la afirmación se presente en una forma («Es» / «Es ist») que en alemán reivindica la generalidad, lo que hace que su condición de validez parezca cuestionable a la luz de las críticas a esas formas de filosofar que Nietzsche formula en la primera sección principal de Más allá del Bien y del Mal ${ }^{21}$.

18 Todas las citas directas que referenciamos a continuación están tomadas de JGB 27 (Nietzsche, cit., 316f.).

Para los lectores que no disponen actualmente de este texto, se lo reproduce aquí en su totalidad: «Es difícil ser comprendido: sobre todo cuando uno piensa y vive gangasrotogati, entre personas que piensan y viven de un modo distinto, a saber, kurmagati o, en el mejor de los casos, mandeikagati, 'conforme a la zancada de la rana' - ¿acaso no estoy haciendo todo lo posible para que resulte difícil comprenderme a mí mismo? - y debemos ser reconocidos de corazón por la buena voluntad para cierta sutileza de interpretación. Ahora bien, en lo que se refiere a 'los buenos amigos', aquellos que siempre están demasiado cómodos y que, precisamente como amigos, creen tener un derecho a la comodidad: uno hará bien en concederles de antemano un campo de juego y una palestra para la malinterpretación: así que uno tiene que reírse; - o eliminarlos por completo, a estos buenos amigos, - iy también reírse!»

19 El original dice «Es ist schwer verstanden zu werden». El verbo alemán verstehen varia en su sentido entre 〈entender) y 〈comprender). En este articulo he optado para usar 〈comprender〉 por tener un significado más amplio que «entender〉 en español. Así corresponde al amplio sentido de verstehen en alemán.

20 Werner Stegmaier («Nietzsches Zeichen», en Nietzsche-Studien 29 (2000), 41-69), debido al hecho de que ya interpreta JGB 27 desde la perspectiva de su comprensión previamente recapitulada de Nietzsche, parte de la premisa opuesta: Nietzsche «no empezó por la comprensión, sino por la no comprensión: Los individuos no podían entenderse como individuos» (ibid., 42).

21 Paradigmáticamente, esta crítica del estado de generalidad de la filosofía anterior tiene lugar en JGB 6, donde ya en la primera frase un yo-narrador identifica «toda gran filosofía» como «la autoconfesión de su autor y una especie de memorias involuntarias y inadvertidas». 
Como también se ha señalado en estudios recientes ${ }^{22}$, las siguientes subfrases ya implementan de manera performativa la tesis de la apertura del texto:

sobre todo cuando uno piensa y vive gangasrotogati, entre personas que piensan y viven de un modo distinto, a saber, kurmagati o, en el mejor de los casos, mandeikagati, "conforme a la zancada de la rana"

La dificultad de comprensión -la que la primera frase afirma como universal- se manifiesta aquí al recurrir a tres términos sánscritos, de los cuales sólo uno se hace explícito. Nietzsche los había anotado en la portada del cuaderno W I $7^{23}$, pero no había recurrido a ellos en los borradores anteriores de JGB $27^{24}$. Así, las frases parciales, que en sí mismas son difíciles de entender, no sólo fundamentan la pretensión de validez de la tesis inicial, sino que, gracias a su forma de presentación («uno») $)^{25}$, también refuerzan su pretensión de validez general. Esto lleva a una primera diferenciación de la tesis articulada inicialmente. Mientras que al principio del texto se habla todavía en general de que es difícil de ser comprendido, ahora se añade una nueva dimensión a la dificultad de comprensión, que podría describirse como fisiológica: Dependiendo de cómo - o, para permanecer en la imagen del texto, a qué velocidad- piensen y vivan los interlocutores en cada caso, el grado de incomprensión cambiará. JGB 27 parece reactivar aquí un topos que ya juega un papel importante en la primera sección principal. Allí se hace referencia varias veces a las condiciones fisiológicas, que subyacen al pensamiento. Lo mismo ocurre respecto del desarrollo de la filosofía de lenguaje en JGB

22 Sommer, Kommentar (cit.), $234 \mathrm{f}$.

23 Como muestra el facsímil, Nietzsche ya había usado la portada para un borrador de título y sólo añadió las palabras en sánscrito después. La notación en sí misma dice: «gangasrotogati "fluyente como la corriente del Ganges" = presto | kurmagati "con el andar de la tortuga" = lento | mandeikagati "con el andar de la rana" = staccato» (KGW IX/4, W I 7, 9).

24 En cuanto a las palabras en sánscrito, existen dos fuentes posibles: Eine Reise nach Ostindien IV de Julius Jolly y el volumen III de la Bibliothek für moderne Völkerkunde, que usa el pasaje de Jolly a este respecto casi palabra por palabra, descuidando las marcas diacríticas (Sommer, Kommentar (cit.), 235f.). Los pasajes están dedicados a los tempi de los cantos indios y, por lo tanto, están orientados a la teoría de la música y la historia cultural. Nietzsche camufla casi completamente este contexto original de los términos en JGB 27 para instrumentalizarlos para en su planteamiento hermenéutico-pragmatista. En relación a esta instrumentalización, cabe señalar que el significado original de las expresiones es irrelevante, ya que Nietzsche trabaja en JGB 27 principalmente con el desconocimiento de las expresiones. Esta forma de descontextualización y recontextualización, así como la consiguiente resemantización de las fuentes, se encuentra frecuentemente en la obra de Nietzsche, pero todavía no ha sido investigada sistemáticamente.

25 El texto alemán recurre al pronombre general «man» $-«[\ldots]$ besonders wenn man gangasrotogati denkt $[. ..] \gg-$, que no se puede traducir literalmente al español por su falta de este tipo de pronombre. 
20, donde se atribuye el «hechizo de determinadas funciones gramaticales» (es decir, el «dominio y [...] la dirección inconsciente de unas funciones gramaticales idénticas») al «hechizo de los juicios de valor fisiológicos y de condiciones raciales» (JGB 20, 310). Si esta reducción en JGB 20 sirve sobre todo para probar genealógicamente el condicionamiento lingüístico de la filosofía, su reactivación intratextual en JGB 27 conduce a una reafirmación de la distinción entre los tipos de seres humanos, cuya reflexión es uno de los leitmotiv de JGB y culmina con la pregunta «¿qué es aristocrático?» que da título a la última sección principal. Directamente relacionado con esta cuestión hay otro topos, que, sin embargo, sólo se comprende si se conoce el significado de los términos sánscritos así como su paralelismo con dos tempi musicales (presto y lento) y una forma de articulación musical (staccato). Esta es la conexión entre, como dice JGB 28, el «tempo medio» del «metabolismo» de una comunidad lingüística y el «tempo de su estilo» y sus consecuencias para el contenido semántico de un texto. Se trata de un topos que JGB discute en varias secciones del texto (JGB 28, 246, 247a). Como ha demostrado Beat Röllin, en abril de 1886 estas secciones pertenecían aún a una misma sección principal de JGB, por entonces intitulada «máscaras», que fue luego abandonada, donde dichas secciones se sucedían de manera inmediata interrumpidas por lo que más tarde se convirtió en JGB $40^{26}$.

Sin embargo, como se demostrará a continuación, JGB 27 tiene un impulso propio, que es, entre otras cosas, consecuencia del hecho de que ahí los términos sánscritos no son definidos. Una contribución significativa a este impulso es el paréntesis que le sigue, el cual contiene una pregunta potencialmente retórica que, en vista de lo que se ha dicho hasta ahora, puede resultar inquietante: « ¿acaso no estoy haciendo todo lo posible para que resulte difícil comprenderme a mí mismo?»

Lo más irritante de este paréntesis es el cambio del modo narrativo. Hasta este punto, el texto trata cuestiones hermenéuticas/pragmatistas desde una posición narrativa ambiguamente marcada. Ahora la narración cambia a la primera persona del singular y por lo tanto a un modo de hablar muy personal. Lo que resulta provocador no es el cambio del modo narrativo en sí mismo (pues JGB se caracteriza desde el principio por una multiplicidad de voces narrativas que contribuyen en última instancia a una forma de filosofar en y desde distintas perspectivas ${ }^{27}$ ), sino las consecuencias que tiene para la interpretación del principio del texto. Recordamos que la frase inicial presenta la dificultad de comprensión como un a priori hermenéutico-pragmático cuasi-objetivo («Es $[. .] »$.$) , de acuerdo con la concepción fisio-gramatical del$

26 Röllin, loc. cit., 54.

27 Born, «Perspektiven» (cit.), 8-11. 
lenguaje de las secciones precedentes. La realización performativa de este «a priori» en las siguientes frases de JGB 27 puede interpretarse, por lo tanto, como su correspondencia textual-inmanente. Pero como el yo-narrador presenta esta realización en dicho paréntesis como una consecuencia potencial de un acto intencional («[yo] estoy haciendo todo lo posible»), el estado de validez general del modelo de comunicación se vuelve cuestionable: ¿es sólo una consecuencia de un acto intencional del yo-narrador y no -como se asumió al principio de JGB 27- un hecho inevitable de la pragmática de la comunicación? ¿Qué significa ello para la pregunta autorreferencial articulada en el paréntesis? ¿Añade simplemente una dimensión intencional al modelo presentado anteriormente o lo socava al confirmarse a sí mismo?

Como muestran estos cuestionamientos, la pregunta autorreferencial conduce en última instancia a la subversión del modelo de comunicación desarrollado hasta ese momento en JGB 27. Este estatus (auto-)subversivo se refuerza por el hecho de que la pregunta es potencialmente retórica: Si uno lo interpreta como tal, esto conduce a la pregunta de por qué la difícil inteligibilidad indicada en el paréntesis no es en sí misma difícil de comprender. Si, por el contrario, uno responde a le pregunta negativamente, su contenido entra en una relación de tensión con la cláusula que le precede, que evidentemente es difícil de entender. Esto significa, sin embargo, que ya en este punto de la interpretación de JGB 27, se puede afirmar que dicho pasaje no sirve como meta-texto central de la supuesta teoría de la comunicación de Nietzsche, si se entiende por teoría una «estructura lingüística que organiza los fenómenos de un área temática en forma propositiva o conceptual» ${ }^{28}$. Esto es así porque su 〈contenido〉 queda en suspenso como autorreferencia debido a la forma de presentación que se acaba de trazar y, por lo tanto, elude una fijación propositiva. En el JGB 27, por lo tanto, la representación de una teoría de la comunicación es reemplazada por la puesta en escena de una práctica de comunicación autosubversiva. Una práctica de comunicación que, debido a su forma autorreferencial, escapa a una representación tética.

A pesar de todo eso, el texto vuelve al modelo desarrollado en las frases que preceden al paréntesis ya en la siguiente subfrase. Se lee: «y debemos ser reconocidos de corazón por la buena voluntad para cierta sutileza de interpretación»

La cláusula inicia un nuevo cambio de modo, que, sin embargo, no es, como en el paréntesis, un cambio de forma narrativa -esta corresponde a la que precede al paréntesis-, sino un cambio en la función ilocutora de lo que se dice: si el comienzo de JGB 27, con excepción del paréntesis, consiste

28 Christian Thiel, «Theorie», en Jürgen Mittelstraß (ed.), Enzyklopädie Philosophie und Wissenschaftstheorie, vol. 4: Sp-Z, Stuttgart: Metzler 2006, 260-270, aquí 260. 
enteramente en declaraciones constitutivas, el texto pasa ahora a un modo normativo («man soll»/《debemos«). La subfrase, cuyo estatus ético ya ha sido señalado por Stegmaier, ${ }^{29}$ parece sacar las consecuencias de lo anterior: Si potencialmente toda la comunicación o el yo-narrador del texto es difícil de comprender, entonces se requiere una cierta forma de afrontar esta situación. La que sugiere el texto («ser reconocidos [...] por la buena voluntad para cierta sutileza de interpretación») es, por un lado, que tal voluntad no puede darse por sentada y, por el otro, que -aunque se dé- sólo se puede esperar «cierta sutileza de interpretación», es decir, en el mejor de los casos una interpretación atenta a los matices de lo dicho y al mismo tiempo estéticamente agradable. También es sorprendente que la referencia sea a la «sutileza de la interpretación» y no a la «sutileza de la comprensión», lo que lleva a la pregunta de cómo se relacionan la «interpretación» y la «comprensión» entre sí. Una mirada a los léxicos del habla alemana contemporánea muestra la proximidad de las dos expresiones, pero sin aclarar si, a pesar de esta proximidad, las diferencias semánticas que parecen caracterizar el uso actual de las expresiones ya estaban presentes en la primera mitad del siglo XIX. Así, el Großes Konversationslexikon de Meyer de 1905 dice bajo el lema «exégesis (interpretación)»:

El descubrimiento y la representación del significado de algo (palabras, signos, etc.), especialmente el significado de un pasaje de las escrituras. [...] La exégesis se afirma como un arte en la filología, donde tiene que transmitir la correcta comprensión de las obras escritas como una interpretación. ${ }^{30}$

Si se compara esta determinación de las dos expresiones con su uso en Más allá del bien y del mal, se hace evidente que Nietzsche -lo que no es sorprendente- también sitúa la expresión 〈interpretación〉 principalmente en el plano filológico, pero la sitúa en una relación diferente con la 〈comprensión〉 a la que alude la entrada del diccionario. Así, por ejemplo, en JGB 22, que critica la interpretación del mundo por parte de los físicos, la expresión sirve para expresar el problemático estado epistemológico de su concepción del mundo: «Pero, como ya he dicho, esto es interpretación, no texto» (JGB 22). Este pasaje, así como el inmediatamente posterior sobre la interpretación de la naturaleza «con una intención y una técnica de interpretación opuestas», sugieren que aquí se hace una distinción entre un texto o su significado y su interpretación con la ayuda de la expresión 〈interpretación〉. De allí que la expresión 〈interpretación〉 denote, no «la correcta comprensión de un texto», como sostiene el Meyers, sino una (re-)semantización impulsada por el interés del intérprete. Este significado se apoya en otros pasajes de Más allá del bien

29 Cf. Stegmaier, «Nietzsches Zeichen» (cit.), 45.

$30 \mathrm{http}: / / \mathrm{www} . w o e r t e r b u c h n e t z . d e / M e y e r s ? l e m m a=$ auslegung 
$y$ del mal (cf. JGB 38, 47, 190), y se corresponde también con las recientes investigaciones sobre la influencia de la educación filológica de Nietzsche en su posterior concepto de interpretación. ${ }^{31}$

En el caso de la frase que aquí se examina, esto significa que con ella no sólo se reafirma la dificultad de comprensión anteriormente señalada, sino que experimenta un significado adicional, ya que ahora incluso la exigencia ética se hace reconocible por «cierta sutileza de interpretación». Sin embargo, si la interpretación ha de entenderse como una (re)semantización guiada por intereses, entonces la exigencia de reconocimiento de «cierta sutileza de interpretación» implica no sólo que esa sutileza rara vez se encuentre, sino también que en la comunicación oral o escrita se suela tratar no sólo de una comprensión difícil, sino de interpretaciones bastante arbitrarias. Así, al final de la frase se establece un concepto de comunicación que, en primer lugar, no procede de la comprensión sino de la malinterpretación (Missverstehen) y que, al mismo tiempo, debido a su forma de presentación, subvierte su propio estatus conceptual al realizarlo de forma autorreferencial.

Con la siguiente frase parece haber un cambio de tema, ya que -al menos a primera vista- sale del marco de la reflexión hermenéutica-pragmatista:

Ahora bien, en lo que se refiere a 'los buenos amigos', aquellos que siempre están demasiado cómodos y que, precisamente como amigos, creen tener un derecho a la comodidad.

La frase está dedicada a una forma característica de comportamiento de los supuestamente «buenos amigos»: su «comodidad». La «comodidad», sin embargo, representa una forma de comportamiento caracterizada por el hecho de que la persona con dicha inclinación rehúya a los obstáculos y al esfuerzo. Tal persona difícilmente reunirá esa «buena disposición» para la «sutileza» en la interpretación de las declaraciones de los demás, que parece requerir una forma de comunicación como la descrita y realizada en la frase anterior, sino que será bastante torpe en su malinterpretación (Missverstehen), especialmente si supuestamente se ve a sí mismo como un «buen amigo» (un auto-entendimiento cuyo estatus es cuestionado por las comillas de Nietzsche) e incluso reclama un «derecho a la comodidad». La siguiente frase saca las consecuencias de este estado de cosas: «uno hará bien en concederles de antemano un campo de juego y una palestra para la malinterpretación»

Si se parte, como se sostiene en la primera parte de JGB 27, de una práctica de comunicación que supone una difícil comprensión y que por lo tanto se caracteriza por la malinterpretación (Missverstehen) -una malinterpretación

31 Cf. Christian Benne, Nietzsche und die historisch-kritische Philologie, Berlín/New York: De Gruyter 2006, especialmente 197-212. 
cuya forma más limitada se caracteriza por la «sutileza de interpretación», es decir, por una (re)semantización que presta atención a los matices-, entonces sólo parece coherente dar a los interlocutores que se caracterizan por el hecho de que no despliegan esas sutilezas por conveniencia ${ }^{32}$, un marco especial de incomprensión, «un campo de juego y una palestra para la malinterpretación». Este marco difiere -al menos así lo sugiere la terminología empleada- de otras formas de malinterpretación en el sentido de que, por una parte, sería más incivilizado, es decir, menos matizado en sus formas de malinterpretación («palestra»/Tummelplatz ${ }^{33}$ ), pero, por otra parte, permitiría la posibilidad de limitar esa malinterpretación al adiestramiento («un campo de juego»/ Spielraum). Si se concede tal marco a alguien, significa que se es consciente de la naturaleza problemática del mismo, es decir, en el caso dado se sabe que los «buenos amigos» lo entienden a uno más torpemente que a los demás.

Si bien estas consecuencias, formuladas como una recomendación, están en armonía con lo que se desarrolló previamente y con lo que se realizó de manera performativa en JGB 27, hay nuevamente una provocación al final de este último texto, cuando se dice: «-así que uno tiene que reírse; -o eliminarlos por completo, a estos buenos amigos, - ¡y también reírse!»

La conexión establecida aquí entre la tendencia a malinterpretar torpemente a los amigos, la concesión de esta tendencia a malinterpretar y la abolición de los supuestos amigos y la risa es en sí mismo difícil de entender. Parece que el texto no es comprensible ${ }^{34}$ o que, en el mejor de los casos, se malinterpreta de forma matizada, por el hecho de que el modelo de comunicación propagado en JGB 27 también se aplica aquí de forma performativa. Esta interacción entre la realización performativa de un supuesto modelo de comunicación y su potencial autosubversión representa la diferencia central entre JGB 27 y los borradores de los que surgió genéticamente el texto finalmente publicado. Una comparación con estos permite perfilar más concretamente el contenido semántico del texto publicado.

Los borradores más antiguos de JGB 27 que se han conservado se encuentran en una doble página en el cuaderno N VII 2 (KGW IX/2, N VII $2,79 \mathrm{f}$. ${ }^{35}$. Nietzsche usó este cuaderno, con interrupciones, durante un largo

32 Laurence Lampert (Nietzsche's Task (cit.), 67) sostiene que por «buenos amigos» hay que entender no los amigos reales de Nietzsche, sino todos los lectores familiarizados con su obra.

33 Cf. http://www.woerterbuchnetz.de/Adelung?lemma=tummelplatz

34 Werner Stegmaier llega a una interpretación similar de esta frase (cf. Stegmaier, «Nietzsches Zeichen» (cit.), 46).

35 Todas las citas siguientes están tomadas de la nueva edición del Nachlass de Nietzsche, la KGW IX, que ofrece las notas manuscritas de Nietzsche como transcripciones diplomáticas. En cuanto a la génesis, estructura y consecuencias interpretativas de esta edición, cf. Martin Endres y Axel Pichler, «"warum ich diesen mißrathenen Satz schuf”. Ways of Reading Nietzsche in the Light of KGW IX» Journal of Nietzsche Studies 44:1 (2013), 90-109. 
período de tiempo, desde agosto de 1885 en adelante. Según Beat Röllin, su primera anotación, escrita con lápiz, data probablemente de principios de 1886. Comparada con el texto publicado, la dicha anotación tiene un carácter mucho más personal. Refiere inmediatamente a la primera persona del singular - «es difícil comprenderme» - y se aleja así de la apertura del texto de JGB 27, que pretende ser general. En esta anotación no se encuentra una justificación de la dificultad de comprensión del yo-narrador. En cambio, la notación saca inmediatamente las consecuencias de ello expresando la intención («y me he propuesto») de dar a los amigos «un amplio campo de juego» o, después de una revisión del texto, la necesidad («y sería un bufón si no») de dar «algún campo de juego para malinterpretar», para expresar la gratitud, conocida de la versión publicada, hacia una «buena disposición para cierta sutileza de interpretación». La relación que se establece de esta manera entre la naturaleza difícil de comprender a un yo-narrado, y la intención de este narrador, de conceder a los amigos un margen para la malinterpretación y agradecer dicha sutileza interpretativa, no tiene las irritantes implicaciones hermenéutico-pragmáticas del texto publicado, ya que lo que se dice se limita a un yo-narrador y a una selección de sus posibles interlocutores, por lo que ser difícil de ser comprendido per se no tiene la pretensión universalmente válida que tiene en la versión publicada.

Sólo la segunda anotación, que se encuentra en la misma página de dicho cuaderno y que, al igual que la primera anotación, muestra rastros de revisión, convierte lo que antes era todavía una articulación muy personal en una general, pero al hacerlo también se mantiene alejada de la concepción de comprensión del texto publicado. La frase inicial allí se lee en la versión de la «capa base» ${ }^{36}$ : «El que es difícil de ser comprendido también lo sabe, tendría que ser un bufón si no dejara al menos a sus amigos un campo de juego para la malinterpretación»

Aquí ya no es un yo el que habla de las consecuencias que saca de la dificultad de ser comprendido, sino que, partiendo de la tesis ya generalizada de que los difíciles de comprender son conscientes de ello, la concesión de un «campo de juego para la malinterpretación» a los amigos se muestra como una expresión de prudencia. Después de esta apertura ya parcialmente generalizadora, la versión borrador se dirige entonces directamente a los amigos en un discurso en primera persona y articula no sólo su gratitud a la «buena disposición para la sutileza de interpretación», sino que también da dos razones más por las que «deja un amplio campo de juego para la

36 En la filología de la edición en alemán y en la investigación del proceso de escritura, la primera versión de un borrador manuscrito, que a menudo se sobrescribe en el curso de su revisión, se denomina «capa base〉 (Grundschicht). 
malinterpretación»: «por coraje» y por el hecho de que la amistad «lo tiene todo muy escondido». Estas razones no están directamente relacionadas con la dificultad de comprensión, por el contrario, la concesión de un «campo de juego para la malinterpretación» se identifica explícitamente como intencional, lo que da lugar a un modelo de comunicación que difiere de la primera versión borrador y de la versión finalmente publicada en que no se establece una relación directa entre la dificultad de comprensión y el malentendido, ni está claro si la comprensión sea posible más allá del grupo de los que son difíciles de ser comprendido.

Ciertas diferencias en el modelo de comunicación también se pueden encontrar en el último borrador que se encuentra en la doble página N VII 2 , 79f. Esta versión ya se ha utilizado en la investigación para la interpretación de JGB $27,{ }^{37}$ ya que es la única de las tres versiones borradores de la doble página que Colli y Montinari han incluido como texto normalizado en su edición de Nietzsche (cf. NL 1[182], KSA 12, 50f.). El texto comienza con esa potencial reivindicación de generalidad, que también abre el JGB 27 - «Es difícil ser comprendido» - pero luego, a diferencia de la versión publicada, se dedica inmediatamente a las consecuencias de la dificultad de comprensión en el trato con los amigos, cuyo estatus tampoco se cuestiona, por lo que la anotación ofrece dos versiones con respecto a esta forma de trato, debido a su revisión por Nietzsche. En la 〈capa base〉, la frase inicial va seguida inmediatamente de la exigencia autoimpuesta de «conceder a sus amigos un campo de juego para la malinterpretación». A continuación, se explican los efectos positivos de esta concesión. ${ }^{38}$ La situación es diferente después de la revisión. También en esta versión faltan tanto la escenificación performativa de la frase inicial mediante la inclusión de palabras en sánscrito (que son realmente difíciles de entender) como la inserción autorreferencial -estos momentos se encuentran exclusivamente en el texto publicado-; en cambio en esta versión, se articula inmediatamente la gratitud por la «buena disposición para cierta sutileza de interpretación» (atribuida no sólo a los amigos) y la concesión de «un amplio campo de juego para la malinterpretación» dado sólo a los amigos. Sin embargo, posteriormente, en esta versión, no sólo se restringe la pretensión de generalidad de ser difícil de ser comprendido, como en todas las anteriores, sino que incluso se habla explícitamente de comprensión: «Creo que es mejor ser malinterpretado que ser incomprendido. ¿Ser comprendido? ¿Sabéis lo que eso significa, no? - Comprendre c'est égaler».

37 Cf. Stegmaier, «Nietzsches Zeichen» (cit.).

38 «En los días buenos uno tiene el placer de hacerlo; sin traicionarlo en los días malos uno encuentra al menos el consuelo así mismo muy escondido» (KGW IX/2, N VII 2, 80). 
Nietzsche distingue aquí entre malinterpretar, ser incomprendido y ser comprendido. Es decir, no excluye aquí la posibilidad de comprensión, que el texto publicado intenta hacer performativamente imposible. Sin embargo, el yo-narrador rechaza la comprensión mediante una cita de los Essais de Paul Bourget -que Nietzsche especifica más adelante en el curso de la revisión del texto $^{39}$ - y la niega por razones de distinción, no porque se base en una teoría de la comunicación que lo guía. Lo mismo pasa con el ser-incomprendido (unverstanden sein), que en alemán es una forma anticuada de no ser comprendido (nicht verstanden werden): «Halaga más ser malinterpretado que ser incomprendido: contra el incomprendido uno se queda frío, y la frialdad insulta»

La malinterpretación se valora psicológicamente por encima de los otros dos modos de comprensión. Sin embargo, esta ultima versión debe una explicación explícita de la relación entre la malinterpretacion y la dificultad de ser comprendido, ya que no explica el fundamento fáctico de los diferentes modos psicológicos de reacción.

En resumen, la comparación contrastada entre las versiones borradores de Nietzsche que preceden a JGB 27 en términos genéticos ${ }^{40}$, muestra que sólo el texto publicado posee el potencial de autosubversión que hace que su modelo de comunicación, que también es mucho más radical que el de sus predecesores, sea en sí mismo cuestionable. Un texto de este tipo no corresponde a una metateoría hermenéutica-pragmática. Sin embargo, un texto así puede producir en sus lectores efectos de los que son responsables las metateorías en textos que no realizan formas performativas de filosofar. En el caso de JGB 27 , estos efectos consistirán probablemente en que se leerán las secciones restantes del libro bajo la sospecha de que se realice en ellas una práctica de comunicación que escenifica la dificultad de ser comprendido y, en el curso de ésta, se autosubvierta potencialmente el modelo de comunicación articulado en paralelo. Un lector de este tipo se abstendrá de extraer precipitadamente contenidos propositivos de estos textos para atribuírselos al autor de Más allá

39 Nietzsche, en el curso de la revisión, amplía la frase de la siguiente manera: «Creo que es mejor ser malinterpretado que ser incomprendido: hay algo insultante en ser compr.» (KGW IX/2, N VII 2, 80).

40 El último borrador, que precedió inmediatamente a la publicación -se conserva en el cuaderno W I 8, 251 (KGW IX/5, W I 8, 251)- no se tratará aquí en detalle, ya que proporciona una versión abreviada del JGB 27 y es casi idéntico a éste. Por lo tanto, no son principalmente las variantes mínimas las que son pertinentes para la interpretación de este proyecto, sino los segmentos de texto que faltan con respecto a la versión publicada, es decir, el pasaje con las palabras en sánscrito, así como la pregunta puesta entre paréntesis. Estos son precisamente los segmentos responsables de la posible autosubversión del texto, lo que subraya aún más su papel central en la versión publicada.

El manuscrito de JGB que Nietzsche mando a su editor y en el que hizo por lo demás numerosos cambios, no muestra más retoques de JGB 27: http://www.nietzschesource.org/DFGA/D-18,17 
del Bien y del Mal como sus enseñanzas filosóficas, sino que tratará de mostrar cierta «sutileza de interpretación».

\section{III}

A continuación se explicarán los presupuestos que implícitamente guiaron la nuestra interpretación de JGB 27. Esta reconstrucción no pretende proyectar los supuestos de nuestra propia actividad interpretativa en una teoría de interpretación completamente explicada y elaborada. El objetivo es más bien esbozar la orientación teórica de la interpretación presentada, a efectos de favorecer la comunicación entre diferentes enfoques teóricos, y así ponerla en discusión, para, en el mejor de los casos, iniciar una discusión largamente esperada sobre los métodos en la investigación de Nietzsche. Así pues, trataré de describir mi propia actividad interpretativa con la ayuda de un vocabulario descriptivo que no sea inmanente a ella, a fin de facilitar así el diálogo con prácticas interpretativas alternativas.

Para determinar los supuestos implícitos de mi interpretación, que se caracteriza en particular por su «proximidad al texto» (es decir, por el hecho de que en el curso de la interpretación se recurrió principalmente a la redacción exacta del texto investigado), es evidente que hay que partir de mi tratamiento del texto investigado: el punto de partida para la interpretación de JGB 27 fue la ubicación del texto dentro de la obra de Nietzsche en su conjunto. Se determinó que el texto era la sección 27 de un libro. La comprensión del objeto de investigación como un libro llevó al hecho de que las declaraciones individuales del autor Nietzsche no se entendían como bloques de construcción de un sistema de declaraciones que abarcaba todos los textos de este autor, sino como declaraciones de un sistema limitado: el «libro». En este contexto, «libro» significa una forma de texto medialmente limitada que comprende un cierto número de signos lingüísticos escritos y coherentes ${ }^{41}$. En consecuencia, la limitación es una primera característica central de lo que se entiende aquí como un «libro». Las otras características implícitamente presupuestas pueden ser determinadas por el escalonamiento del contexto realizado en mi interpretación de JGB 27. El escalonamiento de contextos se refiere a la forma en que, en el curso de la interpretación, se refería a las secciones de texto del «libro〉 y a los intertextos, así como a los contextos externos al texto, como el autor o el sistema de lenguaje contemporáneo ${ }^{42}$.

41 Esta vaga definición sigue el uso cotidiano del lenguaje, según el cual un texto es una «redacción "escrita" de una secuencia coherente de declaraciones» (Duden Online: https://www. duden.de/rechtschreibung/Text_Aeuszerung_Schrift), y complementa esta definición con la dimensión mediática que caracteriza al libro.

42 Al reconstruir los contextos relevantes para la interpretación, sigo la diferenciación hecha por Lutz Danneberg (L. D., «Kontext», en: Harald Fricke (ed.), Reallexikon der deutschen 
Tras ubicar JGB 27 como una sección de texto aislada dentro de Más allá del Bien y del Mal, en el curso de la interpretación, se destacaron más características de JGB 27. Se trataba sobre todo de contextos intratextuales, es decir, por un lado, características formales del libro, como el diseño de las figuras, y por el otro, la determinación de sus topoi centrales, como la crítica de la filosofía dogmática o la crítica del lenguaje. La breve introducción sirvió para seleccionar los contextos intratextuales a los que se recurrió en el curso de la interpretación de JGB 27.

Esta se caracterizó como una lectura frase por frase. Aquí el término 〈lectura〉 no tiene por objeto identificar una práctica interpretativa específica, sino destacar que la interpretación siguió la cronología del texto. En el curso de esta lectura, se atribuyó un marco de significado o un significado concreto a las (sub-)frases individuales o a las expresiones que aparecen en ellas. La atribución de significado se hizo recurriendo a léxicos contemporáneos, así como a la comparación con usos alternativos intra- e intertextuales de las expresiones en cuestión. En el curso de esto, se reactivaron los conocimientos desarrollados previamente en la introducción sobre el diseño de las figuras por Nietzsche, las reflexiones lingüístico-filosóficas y el uso de ciertos términos en Más allá del Bien y del Mal, se comprobó la relevancia de dichos conocimientos previos en relación con el pasaje de texto interpretado y, junto a su constatación, se los utilizó de manera interpretativa.

Este procedimiento corresponde a las normas implícitas de vinculación contextual, que ha seguido la interpretación de JGB 27. El punto de partida fue siempre la redacción exacta del texto a interpretar. Este texto, o expresiones seleccionadas de él, fue definió semánticamente con más detalle mediante una comparación contrastada con pasajes de contextos alternativos. El método de la contextualización utilizado en este proceso siguió un procedimiento similar al de los «pasajes paralelos» (Parallelstellen) tradicionales: además de los léxicos y diccionarios que documentan el significado contemporáneo, los pasajes de texto de la obra de Nietzsche que utilizan las mismas palabras o los pasajes que presumiblemente tienen el mismo objeto de referencia se consideraron pertinentes para perfilar el significado. Estos contextos se clasificaron principalmente por el lugar que ocupan en la obra de Nietzsche: Los contextos intra- e intertextuales. En el caso de estos últimos, también se

Literaturwissenschaft, vol. II, Berlín/New York: De Gruyter 2000, 333-337). Danneberg distingue entre contextos intra-, infra-, inter- y extratextuales, entendiendo por contexto intratextual la «relación de una parte de un texto con otras partes del mismo texto en a) términos temáticos o (sólo) b) términos secuenciales», por contexto infratextual la «relación de un texto o una parte de texto con el texto en su conjunto», por contexto intertextual la «relación de un(a parte de un) texto con a) ciertas clases de textos o b) con otros textos e otras partes de textos» y por contexto extratextual la «relación de un texto con circunstancias no textuales». 
dio preferencia a los «pasajes paralelos» que tienen una conexión genética con el texto investigado por sobre los pasajes que no tienen esa conexión, como los «pasajes paralelos〉 de otras obras o supuestas fuentes de Nietzsche. Las versiones borradores, que tienen una conexión genética con el texto publicado, recibieron un estatus especial en la medida en que ellas mismas (siendo anotaciones manuscritas que documentan un proceso de escritura y no textos terminados), fueron sometidas a una lectura textual concisa, y sólo después fueron comparadas con el texto publicado. Detrás de la práctica de escalonamiento del contexto que acabamos de describir está la presuposición teórica de que las secciones individuales de texto en los libros de Nietzsche poseen potencialmente un contenido estético-semántico individual.

Sobre la base del procedimiento que se acaba de describir, así como del escalonamiento del contexto llevado a cabo en el proceso, se pueden determinar con más detalle las características de lo que se entiende como «libro». Desde el punto de vista formal, un «libro» entendido de esta manera es una entidad autónoma cuyos momentos parciales (es decir, las secciones individuales de texto aisladas tipográficamente) tienen una autonomía semántico-estética parcial. En cuanto a la práctica de interpretación, esto significa, por una parte, que el contenido semántico-estético de las secciones individuales del texto se determinó con más detalle al contrastarlas con secciones similares desde el punto de vista temático del mismo texto, pero, por otra parte, que también se recurrió a contextos externos al mismo texto como el sistema lingüístico contemporáneo de Nietzsche y los significados atribuidos a las expresiones individuales en él, así como a intertextos seleccionados.

A través de la selección de dichos intertextos se puede determinar el tratamiento de la categoría de 〈autor〉. Su función se mantuvo al mínimo en el curso de la interpretación: El 〈autor〉 servía principalmente como soporte metonímico para el contexto general de la obra, así como responsable de la producción y composición textual $\mathrm{y}^{43}$, en segundo lugar, para dar plausibilidad a los contextos inter- y extratextuales elegidos ${ }^{44}$. Aparte de esto, no se hizo

43 En la explicación teórica de la relación entre el autor como productor de textos que recurren parcialmente a formas estéticas de representación y la atribución de significado a esos textos mediante la interpretación, veo el mayor desafío para una teoría de la autoría de los textos de Nietzsche que sea compatible con el enfoque textual, que, debido a su carácter autosubversivo, plantea un gran desafío a los conceptos tradicionales de intencionalidad. Como ha insinuado recientemente Andreas Urs Sommer, el autor-sujeto que se atribuye a los libros de Nietzsche por medio de interpretaciones textuales ya no es el «tipo de sujetos trascendentales o trascendental-pragmáticos que se piensan como productores personificados de ciertas proposiciones y a los que se atribuye (según el principle of charity) una voluntad de coherencia, de coherencia sistémica» (Andreas U. Sommer, Was bleibt von Nietzsche?, Berlín: Duncker \& Humblot 2018, 31f.), como es el caso en la historia tradicional de la filosofía.

44 Como se puede ver aquí, incluso una interpretación textual se refiere inevitablemente a contextos extratextuales, lo que se debe, entre otras cosas, a que el lenguaje de cualquier texto (como 
referencia al autor en el curso de la interpretación. Además, el yo-narrador no se equiparó con la persona histórico-biográfica Friedrich Nietzsche, de acuerdo con las tendencias de la investigación reciente ${ }^{45}$, que, con respecto a Nietzsche, aún deben un elaborado concepto de autoría a nivel de la investigación internacional sobre el concepto de autor ${ }^{46}$.

Los fundamentos de referencia contextual realizados en el curso de la interpretación permiten también sacar conclusiones sobre la comprensión de la composición del libro de Nietzsche publicado en 1886, es decir, sobre las relaciones infratextuales entre las distintas secciones del texto de JGB. Se trata de una comprensión del 〈libro〉 según la cual, a pesar de la supuesta naturaleza limitada del mismo, sus secciones individuales no se entienden como elementos de un conjunto orgánico o sistemático. Así pues, no se presupone que las secciones del texto en que se negocia la misma materia o una materia similar coinciden en la presentación de esa materia, sino más bien que esas secciones se contraponen o delimitan potencialmente unas a otras: La organicidad estética y la coherencia sistemática son así sustituidas por la comprensión del «libro» como la manifestación de un movimiento de pensamiento, cuyos pasos parciales se manifiestan en los segmentos de texto aislados tipográficamente del libro.

Por lo tanto, este enfoque ya representa el resultado de cierto preconocimiento de los aspectos formales de los libros de Nietzsche: Libros como Más allá del Bien y del Mal, cuyos segmentos oscilan entre diferentes tipos de texto como el aforismo, o el ensayo, juegan con los sellos de la comprensión contemporánea de qué significa ser una obra, presuponiéndola así como una hoja de referencia negativa - Andreas Urs Sommer ha llamado a esto «la suspensión de Nietzsche de la forma de libro en forma de libro» ${ }^{47}$. Tal concepción del «libro», incluyendo su referencia negativa a conceptos más

ejemplo los textos filosóficos de Nietzsche) tiene evidentemente su base en el lenguaje normal y coloquial contemporáneo a ellos, con el que siguen relacionados incluso en la desviación.

45 Cf. Katarina Grätz, «"Der Dichter verräth sich in seinen Gestalten” - und bleibt ungreifbar. Nietzsche, Zarathustra und Zarathustras Schatten»: Nietzscheforschung 25(2018), 123-136, especialmente $125-130$ y la literatura citada allí.

46 En los últimos años, el significado del autor se ha discutido con tal intensidad en los estudios literarios en lengua alemana que, mientras tanto, se habla - por analogía con la «muerte del autor»de un «retorno del autor». Los estudios nietzscheanos casi no han prestado atención a estos debates actuales, lo que lleva a que, si se reflexiona sobre el concepto de autor, normalmente se queda muy lejos del nivel de reflexión de los debates actuales en los estudios literarios. Sobre el estado del debate actual en los estudios literarios en lengua alemana, véase: Matthias Schaffrick y Marcus Willand (ed.), Theorien und Praktiken der Autorschaft, Berlin/Boston: De Gruyter 2014; y Fotis Jannidis/Gerhard Lauer/Matias Martinez/Simone Winko (ed.), Rückkehr des Autors. Zur Erneuerung eines umstrittenen Begriffs, Tübingen: Niemeyer 1999.

47 Andreas U. Sommer, «Ein philosophisch-historischer Kommentar zu Nietzsches GötzenDämmerung. Probleme und Perspektiven»: Perspektiven der Philosophie 35 (2009), 45-66, aquí 52. 
tradicionales de la obra, parece subyacer a una interpretación cercana al texto, como la practicada anteriormente.

Esta concepción del «libro» se complementa con presuposiciones específicas del significado y de la teoría de interpretación. La práctica interpretativa parece implicar un modelo de significado según el cual el significado de una expresión resulta de la interacción del uso del lenguaje contemporáneo, el uso intratextual, las inferencias implícitas y el diseño formal. Esto se correlaciona con una comprensión implícita de la interpretación, según la cual la interpretación consiste en atribuir un determinado marco de significado o un significado concreto a una expresión, o texto, examinando las ofertas de significado en los contextos mencionados y concretándolas mediante la yuxtaposición.

En lo que respecta presuposición central de que las distintas secciones de texto de los libros de Nietzsche poseen potencialmente un contenido semántico individual, que puede especificarse mediante la comparación con 〈pasajes paralelos〉, debe señalarse enfáticamente que el procedimiento de comparación contrastante resultante de esta presuposición difiere del procedimiento tradicional de 〈pasajes paralelos en el que no se intenta aclarar los supuestos pasajes oscuros de un texto refiriéndose a «pasajes paralelos», sino que, por el contrario, procede de la posible diferencia entre pasajes de texto idénticos o similares, lo que, sin embargo, no excluye la afirmación de su acuerdo semántico en el curso de la interpretación. Esto significa al mismo tiempo que el conocimiento previo de interpretación que guía la misma sólo asume una función heurística con respecto a las formas de los textos de Nietzsche: Se presupone que el uso de los términos y, por tanto, el significado, varían contextualmente y que los libros de Nietzsche socavan las formas más tradicionales de filosofar, pero tiene que ser constantemente reconfirmado en el curso de las lecturas contrastadas.

Esto tiene consecuencias para la comprensión de la composición de los libros de Nietzsche y, por lo tanto, también para la comprensión de su filosofar expresado en los libros: el modo en lo cual las secciones individuales de un libro se relacionan entre sí siempre se determinará en el curso de las lecturas. Por consiguiente, la interpretación de la composición general de un libro de Nietzsche debería idealmente tener lugar sobre la base de una lectura atenta de todo el texto. Sólo en el curso de tal lectura puede justificarse cuál de las secciones individuales del texto puede entenderse realmente como metareflexiones del filosofar de Nietzsche. Como muestra el ejemplo del JGB 27, no tienen por qué ser necesariamente esas secciones las que, por su temática supuestamente generalizable, den la impresión, en una primera lectura rápida, de que ser tales secciones metafilosóficas. Tal vez tal procedimiento lleve a 
la comprensión de que no hay una filosofía de Nietzsche, sino sólo diferentes formas en las que Nietzsche filosofa ...

\section{REFERENCIA BIBLIOGRÁFICA}

ACAMPORA, Christa Davis y PEARSON, Keith Ansell, Nietzsche's Beyond Good and Evil. A Reader's Guide, London/New York: Continuum 2011.

BENNE, Christian, Nietzsche und die historisch-kritische Philologie, Berlín/New York: De Gruyter 2006.

BORN, Marcus Andreas (ed.), Klassiker Auslegen: Friedrich Nietzsche, „Jenseits von gut und Böse", Berlín/Boston: De Gruyter 2014.

BORN, Marcus Andreas, «Perspektiven auf eine Philosophie der Zukunft in Jenseits von Gut und Böse», en: Marcus A. Born (ed.), Klassiker Auslegen: Friedrich Nietzsche, ,Jenseits von gut und Böse“, Berlín/Boston: De Gruyter 2014, 1-16.

DANNBERG, Lutz, «Kontext», en: Harald Fricke (ed.), Reallexikon der deutschen Literaturwissenschaft, vol. II, Berlín/New York: De Gruyter 2000, 333-337

DELLINGER, Jakob, Situationen der Selbstbezüglichkeit. Studien zur Reflexivität kritischer Denk- und Schreibformen bei Friedrich Nietzsche, tesis doctoral, Universität Wien 2015.

ENDRES, Martin y PICHLER, Axel, «'warum ieh diesen mißrathenen Satz sehuf’. Ways of Reading Nietzsche in the Light of KGW IX.» Journal of Nietzsche Studies 44:1 (2013), 90-109.

GRÄTZ, Katarina, «'Der Dichter verräth sich in seinen Gestalten’ - und bleibt ungreifbar. Nietzsche, Zarathustra und Zarathustras Schatten»: Nietzscheforschung 25 (2018), 123-136.

GRÄTZ, Katarina/KAUFMANN, Sebastian (ed.), Nietzsche zwischen Philosophie und Literatur: Von der Fröhlichen Wissenschaft zu Also sprach Zarathustra, Heidelberg: Winter 2017.

JANIDIS, Fotis/LAUER, Gerhard/MARTINEZ, Matías/Winko, Simone (eds.), Rücker des Autors. Zur Erneuerung eines umstrittenen Begriffs, Tübingen: Niemeyer, 1999.

LAMPERT, Laurence, Nietzsche's Task. An Interpretation of Beyond Good and Evil, New Haven/London: Yale UP 2001.

NIETZSCHE, Friedrich, Más allá del Bien y del Mal, en: F. Nietzsche, Obras completas, ed. de Diego Sánchez Meca. vol. IV: Escritos de Madurez II, Madrid: tecnos, 283-437.

PICHLER, Axel, Philosophie als Text - Zur Darstellungsform der „GötzenDämmerung", Berlín/Boston: De Gruyter 2014.

PICHLER, Axel: «Prosopopeisches Denken. Eine textgenetische Lektüre der Vorrede zur Neuauflage der ,Fröhlichen Wissenschaft'», en: K. Grätz/S. Kaufmann (ed.), Nietzsche zwischen Philosophie und Literatur: Von der Fröhlichen Wissenschaft zu Also sprach Zarathustra, Heidelberg: Winter 2017, 29-73.

RÖLLIN, Beat, «Ein Fädchen um's Druckmanuskript und fertig? Zur Werkgenese von Jenseits von Gut und Böse», en: Marcus A. Born y Axel Pichler (ed.) Texturen 
des Denkens. Nietzsches Inszenierung der Philosophie in Jenseits von Gut und Böse, Berlín/Boston: De Gruyter 2013, 47-67.

SCHAFFRICK, Matthias y WILLAND, Marcus (eds.), Theorien und Praktiken der Autorschaft, Berlin/ Boston: De Gruyter, 2014.

SOMMER, Andreas Urs, «Ein philosophisch-historischer Kommentar zu Nietzsches Götzen-Dämmerung. Probleme und Perspektiven»: Perspektiven der Philosophie 35 (2009), 45-66.

SOMMER, Andreas Urs, Kommentar zu Nietzsches Jenseits von Gut und Böse, Berlín/ Boston: De Gruyter 2016 (= Historischer und kritischer Kommentar zu Friedrich Nietzsches Werken, vol. 5/1).

SOMMER, Andreas Urs, Was bleibt von Nietzsche?, Berlín: Duncker \& Humblot 2018. STEGMAIER, Werner, Friedrich Nietzsche zur Einführung, Hamburg: Junius 2011. STEGMAIER, Werner, «Nach Montinari. Zur Nietzsche-Philologie»: NietzscheStudien 36 (2007), 80-94.

STEGMAIER, Werner, Nietzsches Befreiung der Philosophie. Kontextuelle Interpretation des V. Buches der „Fröhlichen Wissenschaft“, Berlín/Boston: De Gruyter 2012.

STEGMAIER, Werner, «Nietzsches Zeichen»: Nietzsche-Studien 29 (2000), 41-69. THIEL, Christian, «Theorie», en: Jürgen Mittelstraß (ed.), Enzyklopädie Philosophie und Wissenschaftstheorie, vol. 4: Sp-Z, Stuttgart: Metzler 2006, 260-270.

ZITTEL, Claus, «Der Dialog als philosophische Form bei Nietzsche»: NietzscheStudien 45 (2017), 81-112. 